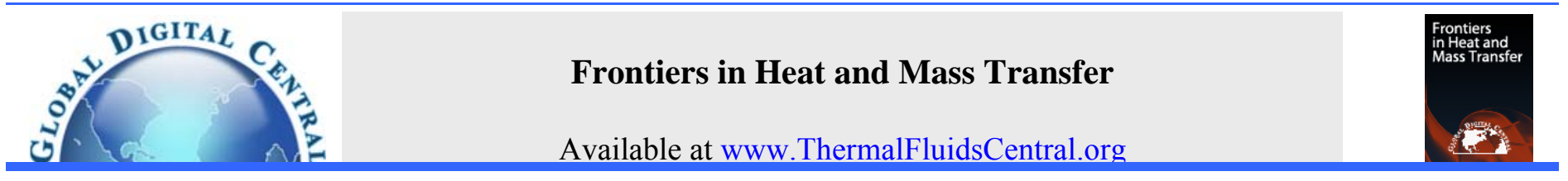

\title{
A COMPARATIVE STUDY OF THERMAL RADIATION EFFECTS ON MHD FLOW OF NANOFLUIDS AND HEAT TRANSFER OVER A STRETCHING SHEET
}

\author{
T. Sravan Kumar, B. Rushi Kumar*
}

Department of Mathematics, School of Advanced Sciences, VIT University, Vellore, TN, 632014, India

\begin{abstract}
In this work, the steady natural convective boundary layer flow of nanofluid and heat transfer over a stretching sheet in the presence of a uniform transverse magnetic field is investigated. We consider two different base fluids and three different nanoparticles were examined as nanofluid. A new model was used in the simulation of nanofluid. Similarity transformations are used to obtain a system of nonlinear ordinary differential equations. The resulting equations are solved numerically by shooting method with Runge-Kutta fourth order scheme (MATLAB package). The effects of various parameters describing the transport in the presence of thermal radiation, buoyancy parameter, magnetic parameter and heat source/sink and nanoparticle volume concentration on the nanofluid velocity, temperature, the heat transfer coefficient and skin-friction coefficient are studied through graphs and table. Furthermore, comparisons with published results are in very good agreement.

Keywords: $M H D$, nanofluids, free convection, thermal radiation, heat source/sink, stretching sheet.
\end{abstract}

\section{INTRODUCTION}

Nanomaterials are being applied in more and more fields with potential applications in the areas of material sciences, electronics, and medicine. Among the nanotechnologies, nanofluid has received great attention in recent years because of its unique properties. Nanofluid is characterized as a base fluid with suspended solid nanoparticles of tiny size $(1-100 \mathrm{~nm})$ and nanofluid exhibits higher thermal conductivity and convective heat transfer coefficient compared to the traditional fluids such as oil, water, and ethylene glycol. As indicated by Choi (1995), the thermal conductivity of the conventional heat transfer fluid improved around two times when included with small nanoparticles even if the fraction is less than $1 \%$ of the volume. Because of enhanced heat transfer characteristics, nanofluids find numerous applications in engineering processes, particularly in the cooling technologies.

Eastman et al. (1997) suggested that an enlargement in thermal conductivity of around $60 \%$ can be accomplished for a nanofluid including water and $5 \%$ vol. of $\mathrm{CuO}$ nanoparticles. This means that the expansion in the surface region owing to the suspension of nanoparticles. Similarly, it was absolutely spoken to that a nanofluid, as well as $\mathrm{Cu}$ nanometer-sized particles scattered in ethylene glycol, has an abundant higher effective thermal physical phenomenon than either for real oil or ethylene glycol having a similar volume fraction of scattered base metal nanoparticles. Eastman et al. (2000) displayed that an improvement in the thermal conductivity relies on the shape, estimate and thermal attributes of nanoparticles. Mechanisms of heat flow in suspensions of nano-sized particles studied by Keblinski et al. (2002). Das et al. (2003) examined a two to a four fold rise in thermal conductivity development for nanofluid containing $\mathrm{TiO}_{2}$ - water or $\mathrm{Al}_{2} \mathrm{O}_{3}$ - water nanoparticles over a small temperature vary from $21^{\circ}-51^{\circ} \mathrm{C}$. To study the effects of the concentration and size variation of the nanoparticles, the concentration and size are varied from $0 \%-5 \%$ and $25-100 \mathrm{~nm}$ respectively over the
Reynolds number range of 250 -1500 for Au -water nanofluid. Buongiorno (2005) analyzed the flow characteristics of viscous, with incompressible fluids with suspended nano-sized solid particles high significant due to the application of such fluids in heat transfer devices. Xie et al. (2010) have examined magnesium oxide nanofluids; higher thermal conductivity and lower viscosity with ethylene glycol-based nanofluids containing oxide nanoparticles. Electro-oxidation of ethylene glycol has attracted considerable interest for mobile, stationary and portable applications owing to its high theoretical energy capacity, high boiling point and high efficiency of electric power conversion has been analyzed in a review article by Yue et al. (2012). The existing literature was shown that ensures the enlargement of nanoparticles in the base fluid may accomplish an essentially reducing in the heat transfer; for comprehensive review, see (Kakac and Pramuanjaroenkij (2009), Sheikholeslami et al. (2014), Nayak et al. (2017), and Nayak et al. (2017)).

The study of boundary layer flow passing a stretching sheet become an important and interesting challenge for research studies due to its practical utility in industry and engineering. It has potential applications in many areas such as cooling of metallic sheet, stretching of the plastic film, industrialized polymer sheet, metal spinning, crystal growing, electronic chips, filaments and wires, glass blowing, artificial fibers, paper production, metallurgical processes, rubber sheets, and polymer extrusion. The quality yet ultimate production formations among these techniques are dependent over the concerning cooling and stretching. The studies on boundary layer flow of nanofluid over a stretching sheet have attracted the attention of a large of a number of researchers. The boundary layer flow and heat transfer in a viscous fluid contacting metallic nanoparticles over a stretching sheet in the presence of thermal radiation have investigated by Hamad and Ferdows (2012). Effects of chemical reaction on the MHD flow of a visco-elastic fluid through porous medium have been presented by Nayak et al. (2014).

*Corresponding author. Email: rushikumar@,vit.ac.in 
Sheikholeslami et al. (2014) have examined the effect of thermal radiation on a magnetic field effect on $\mathrm{CuO}$-water nanofluid and heat transfer. Tukyilmazoglu (2014) have described the exact analytical solution for heat and mass transfer of magnetohydrodynamic slip flow in nanofluids. Unsteady radiative MHD free convective flow and mass transfer of a viscoelastic fluid past an inclined porous plate analyzed by Nayak et al. (2015). Rushi Kumar et al. (2015) investigated on unsteady free convection flow in the presence of magnetic field fixed relative to the fluid or the plate by using Laplace transformation technique. Gireesha et al. (2016) have analyzed the development of steady boundary layer flow of a nanofluid over a stretching sheet in the presence of radiation effects. It is found that the induced magnetic field and temperature distributions are enhanced with the strengthening of hydromagnetic field. Recently Mabood et al. (2016) have studied the MHD stagnation point of water-based nanofluid in which the heat and mass transfer includes the effects of the volume fraction of nanoparticles, radiation, viscous dissipation and chemical reaction.

The investigation of magnetohydrodynamics with heat transfer within the effect of thermal radiation has gained a great consideration due to its diversified applications involved in designs of the fins, steel rolling, manufacturing engineering and various propulsion devices for aircraft, in cooling of reactors in geophysics and astrophysicist. It is associated with the study of solar structures, radio spread by the ionosphere etc. It is because of the interaction of electromagnetic fields and electrically conducting fluids. Conducting fluid moves through the magnetic field, an electric field and therefore a current may be started, and in this manner, the current interacts with the magnetic field to make a body force on the fluid. Such interactions occur both in nature and in new man-made devices. In the research center, numerous devices have been made based on the principle of the magnetohydrodynamic interaction directly, such as impetus units and power generators or which include liquid electromagnetic field interactions; for example, electrical discharges, MHD pumps, electron beam dynamics, MHD bearing, traveling wave tubes, etc. Recently, many researchers have examined the influences of electrically conducting nanofluids, such as water mixed with a petite acid and another ingredient in the presence of a magnetic field on the flow and heat transfer of an incompressible, viscous, electrically conducting fluid past a moving surface or a stretching plate in the motionless fluid. Effects of thermal radiation on the steady laminar MHD boundary layer flow of a nanofluid over an exponentially stretching sheet have been presented by Loganathan and Vimala (2013). Khan (2013) studied the effects of magnetic field on the radiative flow of a nanofluid past a stretching sheet. Sheikholeslami and Ganji (2014) have studied the effects of thermal radiation on unsteady nanofluid flow and heat transfer in presence of magnetic field. Hayat et al. (2014) analyzed the magnetohydrodynamic boundary layer flow of nanofluid. Nayak (2015) examined the chemical reaction effect on MHD viscoelastic fluid over a stretching sheet through a porous medium. Satya Narayana and Venkateswarlu (2016) examined heat transfer analysis of water-based nanofluid over a stretching sheet using different types of nanoparticles such as $\mathrm{Cu}, \mathrm{Ag}, \mathrm{TiO}_{2}$, and $\mathrm{Al}_{2} \mathrm{O}_{3}$. There have been published several recent numerical studies on the modeling of heat transfer and nanofluids (Khan and Pop (2010), Makinde and Aziz (2011), Nadeem et al. (2014), A Daniel (2015), and Pourmehran et al. (2016)).

The objective of the present study is to investigate the MHD natural convective boundary layer flow of nanofluid and heat transfer over a stretching sheet in the presence of heat source parameter. Three types of water; ethylene glycol $50 \%$ based nanofluids containing nanoparticles of copper $(\mathrm{Cu})$, copper oxide $(\mathrm{CuO})$ and magnesium oxide $(\mathrm{MgO})$ have been considering in the present work. This model has considered a new micro-convection model, namely Patel model for more heat transfer capability of nanofluids. A new model was used in the simulation of nanofluid. The governing nonlinear partial differential equations are transformed into a system of nonlinear ordinary differential equations using similarity transformation and then tackled numerically by shooting method with Runge-Kutta fourth order scheme (MATLAB package). The dependency of velocity, temperature, and nanoparticles volume fraction profiles as well as the skin-friction coefficient and local Nusselt number on these parameters has been discussed.

\section{MATHEMATICAL ANALYSES}

The physical model and geometry of the problem are shown in Fig. 1. We consider the two-dimensional steady magnetohydrodynamic boundary layer flow of an incompressible nanofluid over a stretching sheet in the presence of a uniform transverse magnetic field. It is assumed that the magnetic Reynolds number insignificant in the free convection flow, hence the induced magnetic field is ignored. Electric field and dissipation effects are neglected. The $X$-axis is taken along the stretching surface in the upward direction, $y$ - axis is normal to it. A uniform magnetic field of strength $B_{0}$ is applied normal to the fluid flow direction. The nanofluids consist of two different types of base fluids; water, ethylene glycol $50 \%$ with three different types of nanoparticles; namely $\mathrm{Cu}$ (copper), $\mathrm{CuO}$ (copper oxide), and $\mathrm{MgO}$ (magnesium oxide). It is assumed that the base fluid and the nanoparticles are in thermal equilibrium and no slip occurs between them. Under the above assumptions, the boundary layer equations governing for this problem can be written as follows [Rashidi et al. (2014)]

Table 1 Thermophysical properties of water and nanoparticles at $\mathrm{T}=300 \mathrm{~K}$ ([Hakan et al. (2008), Sekulić et al. (2005)]).

\begin{tabular}{|l|l|l|l|l|l|}
\hline $\begin{array}{l}\text { Physical } \\
\text { properties }\end{array}$ & $\begin{array}{l}\text { Pure } \\
\text { water }\end{array}$ & $\begin{array}{l}\text { Ethylene } \\
\text { glycol 50\% }\end{array}$ & $\mathrm{Cu}$ & $\mathrm{CuO}$ & $\mathrm{MgO}$ \\
\hline$\rho\left(\mathrm{kg} / \mathrm{m}^{3}\right)$ & 997.1 & 1052.1 & 8933 & 6320 & 3580 \\
\hline$c_{p}(\mathrm{~J} / \mathrm{kg} . \mathrm{K})$ & 4179 & 3301.7 & 385 & 531.8 & 960 \\
\hline$k(\mathrm{~W} / \mathrm{m} . \mathrm{K})$ & 0.613 & 0.432 & 401 & 76.5 & 48.4 \\
\hline$\beta \times 10^{5} K^{-1}$ & 21 & 57 & 1.67 & 1.80 & 1.26 \\
\hline$\mu_{f}\left(\mathrm{Ns} / \mathrm{m}^{2}\right)$ & $\begin{array}{c}0.001 \\
003\end{array}$ & 0.0031871 & - & - & - \\
\hline$d_{f}$ or $_{s}(\mathrm{~nm})$ & 0.24 & 0.32 & $30-60$ & $30-60$ & $30-60$ \\
\hline
\end{tabular}

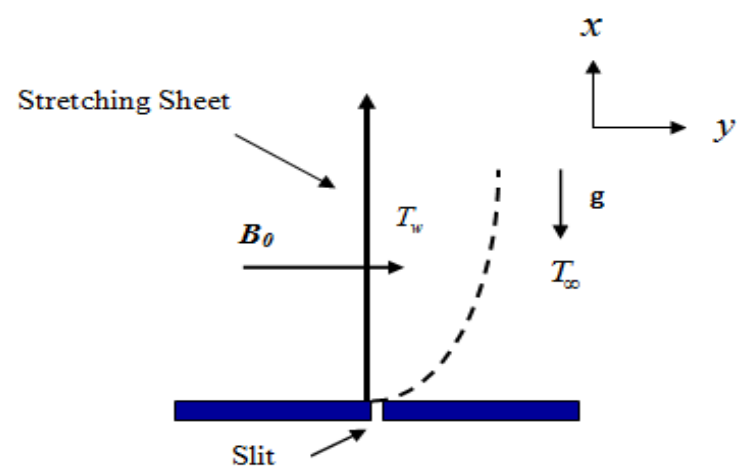

Fig. 1 Physical model

The flow is governed by the following equations:

$$
\begin{aligned}
& \frac{\partial u}{\partial x}+\frac{\partial v}{\partial y}=0 \\
& \rho_{n f}\left(u \frac{\partial u}{\partial x}+v \frac{\partial u}{\partial y}\right)=\mu_{n f} \frac{\partial^{2} u}{\partial y^{2}}+g(\rho \beta)_{n f}\left(T-T_{\infty}\right)-\sigma B_{0}^{2} u \\
& \left(\rho c_{p}\right)_{n f}\left(u \frac{\partial T}{\partial x}+v \frac{\partial T}{\partial y}\right)=k_{n f} \frac{\partial^{2} T}{\partial y^{2}}-\frac{\partial q_{r}}{\partial y}-Q_{0}\left(T-T_{\infty}\right)
\end{aligned}
$$

$u$ is the velocity components along the $x$-direction, $T$ the temperature of the nanofluid, $\mu_{n f}$ the dynamic viscosity of the nanofluid, $\beta_{n f}$ the thermal expansion coefficient of the nanofluid, $\rho_{n f}$ the density of the nanofluid, $k_{n f}$ the thermal conductivity of the nanofluid, $\left(\rho c_{p}\right)_{n f}$ the 
heat capacitance of the nanofluid, Qo heat generation constant, $g$ the acceleration due to gravity and $q_{r}$ the radiative heat flux which are given as in Ref.[ Choi (2001), Pak and Young (1998), Govindaraju et al. (2015)]

$\mu_{n f}=\mu_{f}\left(1+39.11 \phi+533.9 \phi^{2}\right), \rho_{n f}=(1-\phi) \rho_{f}+\phi \rho_{s}$,

$\left(\rho c_{p}\right)_{n f}=(1-\phi)\left(\rho c_{p}\right)_{f}+\phi\left(\rho c_{p}\right)_{s},(\rho \beta)_{n f}=(1-\phi)(\rho \beta)_{f}+\phi(\rho \beta)_{s}$

where, $\phi$ is the solid volume fraction of the nanoparticle. The effective thermal conductivity of nanofluid is calculated by Patel et al. (2005) model as follows:

$\frac{k_{n f}}{k_{f}}=1+\frac{k_{s} A_{s}}{k_{f} A_{f}}+c k_{s} P e \frac{A_{s}}{k_{f} A_{f}}, \frac{A_{s}}{A_{f}}=\frac{d_{f}}{d_{s}} \frac{\phi}{1-\phi}, P e=\frac{u_{s} d_{s}}{\alpha_{f}}, u_{s}=\frac{2 k_{b} T}{\pi \mu_{f} d_{s}^{2}}$,

$c=25,000$

Where, $\rho_{f}$ the density of the base fluid, $\rho_{s}$ the density of the nanoparticle, $k_{f}$ the thermal conductivity of the base fluid, $k_{s}$ the thermal conductivity of the nanoparticle, $P e$ the Peclet number, $\alpha_{f}$ the thermal diffusivity of liquid, $\mu_{f}$ the viscosity of the base fluid, $\left(\rho c_{p}\right)_{f}$ the heat capacitance of the base fluid and $\left(\rho c_{p}\right)_{s}$ the heat capacitance of nanoparticles and $\mathrm{c}$ is constant.

The radiative heat flux for an optically thick fluid can be found from Rosseland (1931) approximation and its formula is derived from the diffusion concept of radiative heat transfer in the following way

$q_{r}=-\frac{4 \sigma^{*}}{3 k_{n f}^{*}} \frac{\partial T^{4}}{\partial y}$

where $\sigma^{*}\left(=5.67 \times 10^{-8} \mathrm{~W} / \mathrm{m}^{2} \mathrm{~K}^{4}\right) k^{*}\left(\mathrm{~m}^{-1}\right)$ states 'Stefan-Boltzmann' constant, the Rosseland mean absorption coefficient respectively. It is supposed that due to variation in temperature in the fluid flow domain are adequately very small and that $T^{4}$ may well derive as a linear function of temperature. Hence, this attained by elucidating $T^{4}$ in a Taylor series about $T_{\infty}$, thus:

$T^{4}=T_{\infty}^{4}+4 T_{\infty}^{3}\left(T-T_{\infty}\right)+6 T_{\infty}^{2}\left(T-T_{\infty}\right)^{2}+--$

Ignoring higher order terms in Eq. (6) beyond the first order in $\left(T-T_{\infty}\right)$, we get

$T^{4} \cong T_{\infty}^{4}+4 T_{\infty}^{3}\left(T-T_{\infty}\right)$

$T^{4} \cong T_{\infty}^{4}+4 T T_{\infty}^{3}-4 T_{\infty}^{4}$

$T^{4} \cong 4 T T_{\infty}^{3}-3 T_{\infty}^{4}$

Considering the above equation, Eq. (3) becomes

$\left(\rho c_{p}\right)_{n f}\left(u \frac{\partial T}{\partial x}+v \frac{\partial T}{\partial y}\right)=\left(k_{n f}+\frac{16 \sigma^{*} T_{\infty}^{3}}{3 k_{n f}^{*}}\right) \frac{\partial^{2} T}{\partial y^{2}}-Q_{0}\left(T-T_{\infty}\right)$

The boundary conditions of equations (1) to (3) are as follows

$$
\begin{array}{cccc}
u=u_{w}(x)=a x, & v=0, T=T_{w} & \text { at } & y=0 \\
u \rightarrow 0, & T \rightarrow T_{\infty}, & \text { as } & y \rightarrow \infty
\end{array}
$$

The following similarity variables are also introduced

$$
u=a x f^{\prime}(\eta), v=-\sqrt{a v_{f}} f(\eta), \theta(\eta)=\frac{T-T_{\infty}}{T_{w}-T_{\infty}}, \eta=\sqrt{a / v_{f}} y .
$$

We get the following governing equations which are dimensionless.

$$
\begin{aligned}
& f^{\prime \prime \prime}-A a_{1}\left(\left(f^{\prime}\right)^{2}-f f^{\prime \prime}\right)-M A f^{\prime}+\lambda A a_{2} \theta=0 \\
& \theta^{\prime \prime}+\frac{3 N \operatorname{Pr} a_{3} k_{f}}{k_{n f}(3 N+4)} f \theta^{\prime}-\frac{3 N \operatorname{Pr} k_{f}}{k_{n f}(3 N+4)} \theta=0
\end{aligned}
$$

The corresponding boundary conditions become

$$
\begin{aligned}
& f=0, \quad f^{\prime}=1, \quad \theta=1 \quad \text { at } \quad \eta=0 \\
& f^{\prime} \rightarrow \infty, \quad \theta=0 \quad \text { as } \quad \eta \rightarrow 0
\end{aligned}
$$

where

$$
\begin{aligned}
& a_{1}=\left[(1-\phi)+\phi\left(\frac{\rho_{s}}{\rho_{f}}\right)\right], a_{2}=\left[(1-\phi)+\phi \frac{(\rho \beta)_{s}}{(\rho \beta)_{f}}\right], \\
& a_{3}=\left[(1-\phi)+\phi \frac{\left(\rho c_{p}\right)_{s}}{\left(\rho c_{p}\right)_{f}}\right],
\end{aligned}
$$

The dimensionless constants appearing in equations (10) and (11) are the magnetic parameter $M$, buoyancy parameter $\lambda$, viscosity ratio $A$, Prandtl number $\operatorname{Pr}$, heat source parameter $Q$, and $N$ is the radiation parameters which are defined as follows.

$$
\begin{aligned}
& M=\frac{\sigma B_{0}^{2}}{a \rho_{f}}, \lambda=\frac{g \beta_{f}\left(T_{w}-T_{\infty}\right)}{a u_{w}}, A=\frac{\mu_{f}}{\mu_{n f}}, \operatorname{Pr}=\frac{v_{f}\left(\rho \mathrm{C}_{\mathrm{p}}\right)_{f}}{k_{f}}, Q=\frac{Q_{0}}{a\left(\rho C_{p}\right)_{f}}, \\
& N=\frac{k_{n f} k_{n f}^{*}}{4 \sigma^{*} T_{\infty}^{3}}
\end{aligned}
$$

\section{Skin-friction:}

From velocity field, we study the skin-friction which is given in dimensionless form as follows:

$$
C_{f} \operatorname{Re}_{x}^{\frac{1}{2}}=-\frac{1}{A}\left[\frac{\partial u}{\partial \eta}\right]_{\eta=0}=-\frac{1}{A} f^{\prime \prime}(0)
$$

\section{Nusselt Number:}

From temperature field, we study the Nusselt number which is given in dimensionless form as follows:

$N u_{x} \operatorname{Re}_{x}^{-\frac{1}{2}}=-\frac{k_{n f}}{k_{f}}\left[\frac{\partial \theta}{\partial \eta}\right]_{\eta=0}=-\frac{k_{n f}}{k_{f}} \theta^{\prime}(0)$

Table 2 Comparison between the present solutions for various values of $\phi$ with previously published results when $\operatorname{Pr}=6.2, N=Q=\lambda=0$

\begin{tabular}{|l|l|l|l|l|l|}
\hline \multirow{3}{*}{1} & $\phi$ & \multicolumn{2}{|c|}{$\begin{array}{c}\text { Hamad (2011) } \\
(\mathrm{Cu}-\text { water) }\end{array}$} & \multicolumn{2}{c|}{$\begin{array}{c}\text { Present Results } \\
(\mathrm{Cu}-\text {-water })\end{array}$} \\
\cline { 3 - 6 } & & $-f^{\prime \prime}(0)$ & $-\theta^{\prime}(0)$ & $-f^{\prime \prime}(0)$ & $-\theta^{\prime}(0)$ \\
\cline { 2 - 6 } & 0.05 & 1.4524 & 1.5237 & 1.45243 & 1.5236 \\
& 0.1 & 1.4657 & 1.3884 & 1.45842 & 1.3882 \\
& 0.2 & 1.4331 & 1.1670 & 1.43301 & 1.1653 \\
\hline
\end{tabular}

\section{RESULT AND DISCUSSIONS}

The equations (10) and (11) with the boundary condition (12) were solved numerically by shooting method with Runge-Kutta fourth order scheme (MATLAB package). The impacts of various governing dimensionless parameters are examined, namely the magnetic parameter $(M)$, buoyancy parameter $(\lambda)$, radiation parameter $(N)$, volume fraction parameter $(\phi)$, heat source/sink parameter $(Q)$, Prandtl number $(\operatorname{Pr})$ in transit of flow field, transverse velocity $f^{\prime}(\eta)$, temperature $\theta(\eta)$ are studied graphically shown in Figs. 2-9. The values of the volume fraction of nanoparticles are taken in the range of $0 \leq \phi \leq 0.2$.

The behavior of velocity and temperature by the influence of nanoparticles, magnetic parameter, buoyancy parameter, radiation parameter, heat source/sink parameter and volume fraction are illustrated in Figs. 2-9. In Fig. 2 reveals that the effect of volume fraction of nanoparticles on the fluid velocity, it increases due to the absence of surface tension forces and hence, the momentum boundary layer thickness increases. The higher value of volume fraction obtained for CuO-ethylene glycol 50\% than MgO-ethylene glycol 50\% can be attributed to the higher value of copper ionic radius $(0.121 \mathrm{~nm})$ than the magnesium ionic radius $(0.072 \mathrm{~nm})$. From Fig. 3 , it is observed that the effect of volume fraction of nanoparticles of the temperature distribution metallic nanoparticles has much higher heat conductivity than common liquids. It also observed that with the increasing volume fraction of nanoparticles the thermal boundary layer increases. 
In Fig. 4 depicts that the velocity profiles for the various magnetic parameter. It is observed that an increase in magnetic parameter the velocity decreases. It is due to the fact that the application of transverse magnetic field will result in a Lorentz force similar to drag force, which tends to resist the fluid flow and thus reducing its velocity and it is also noticed that the momentum boundary layer thickness increases with increasing value of the magnetic parameter. Physically it is seen that when any fluid is subjected to a magnetic field than the viscosity rises. The results of which is that the fluid's capacity to transfer force can be restricted with help of an electromagnet which gives rise to its various possible control-based applications including MHD ion propulsion, electromagnetic tossing of meats, MHD control time etc. The different values of the magnetic parameter obtained for $\mathrm{CuO}$ - ethylene glycol $50 \%$ and $\mathrm{MgO}$ - ethylene glycol $50 \%$ can be attributed to the higher value of the magnetic moment of copper ions than that of the magnetic moment of non-magnetic magnesium ions.

From Fig. 5 represents the variation of the nanofluid temperature of the magnetic parameter. It is observed that an increase in magnetic parameter the temperature increases. Owing to the nanofluid has thick thermal boundary layer and also, temperature increments in the nanofluid due to its high thermal conductivity. The temperature distribution of $\mathrm{CuO}$ - ethylene glycol $50 \%$ is greater than that of $\mathrm{MgO}$ - ethylene glycol $50 \%$. This can be attributed to the higher value of the magnetic moment of copper ions than that of the magnetic moment of non-magnetic magnesium ions on applying the increasing value of magnetic field. The higher magnetic dipole moment occurred due to an increase of magnetic field in case of copper ions. This, in turn, increases the higher temperature distribution than the magnesium ions.

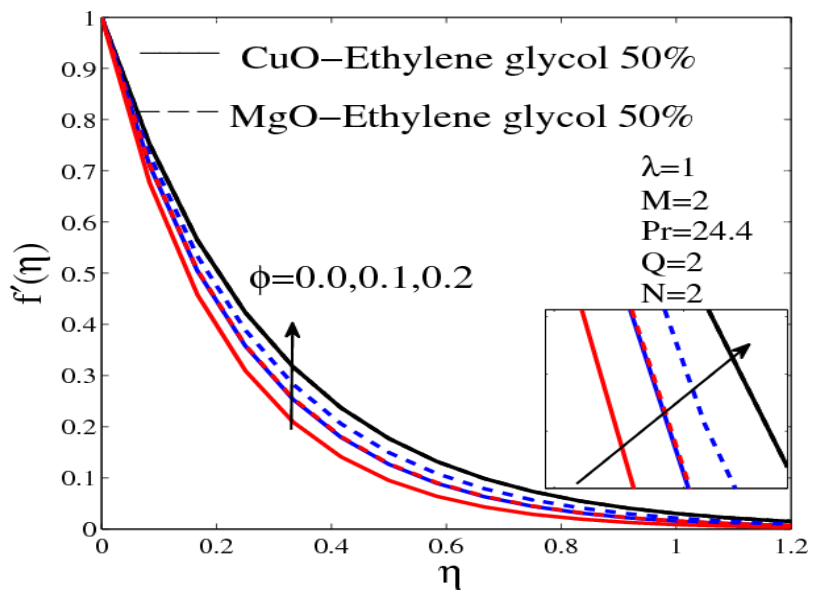

Fig. 2 Velocity profile for different $\phi$

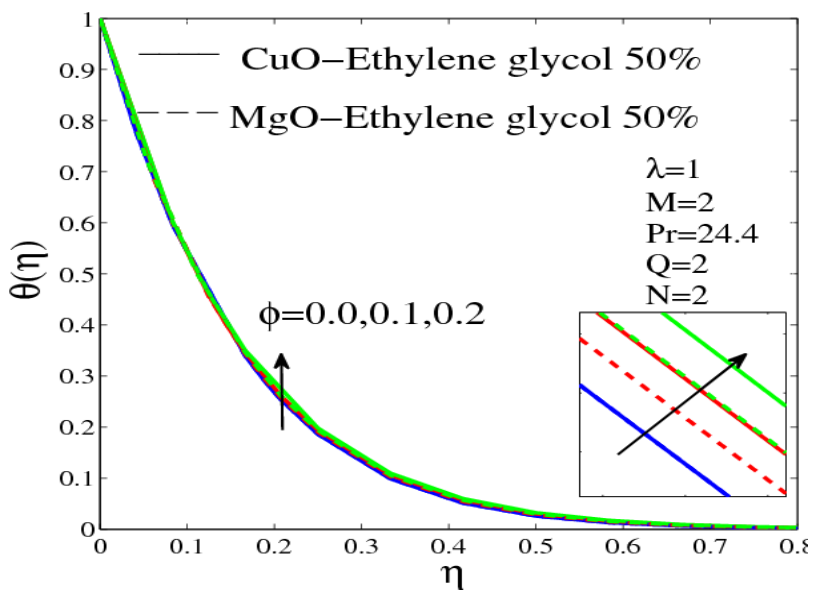

Fig. 3 Temperature profile for different $\phi$

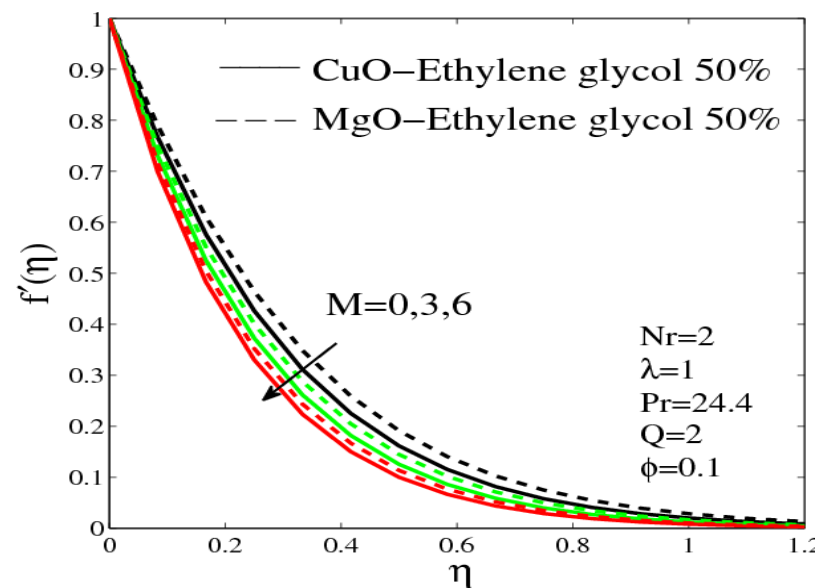

Fig. 4 Velocity profile for different $M$

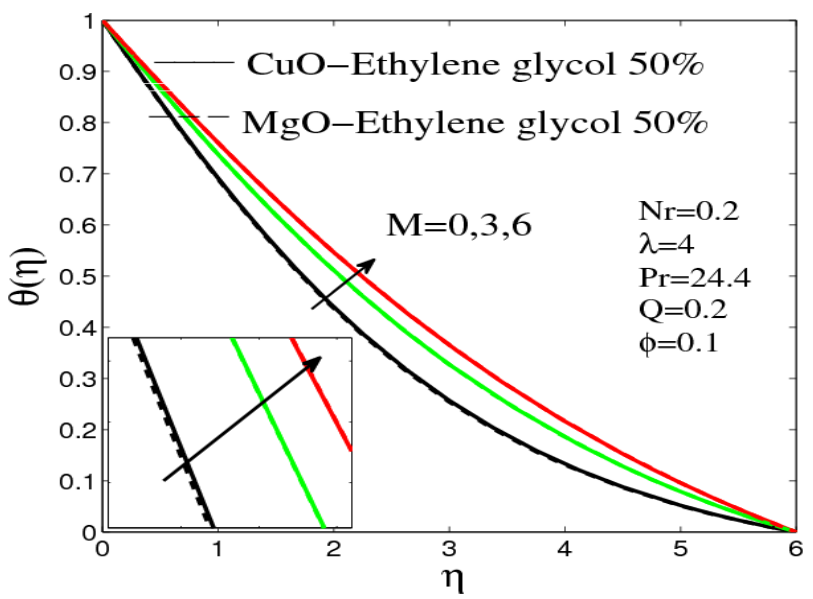

Fig. 5 Temperature profile for different $M$

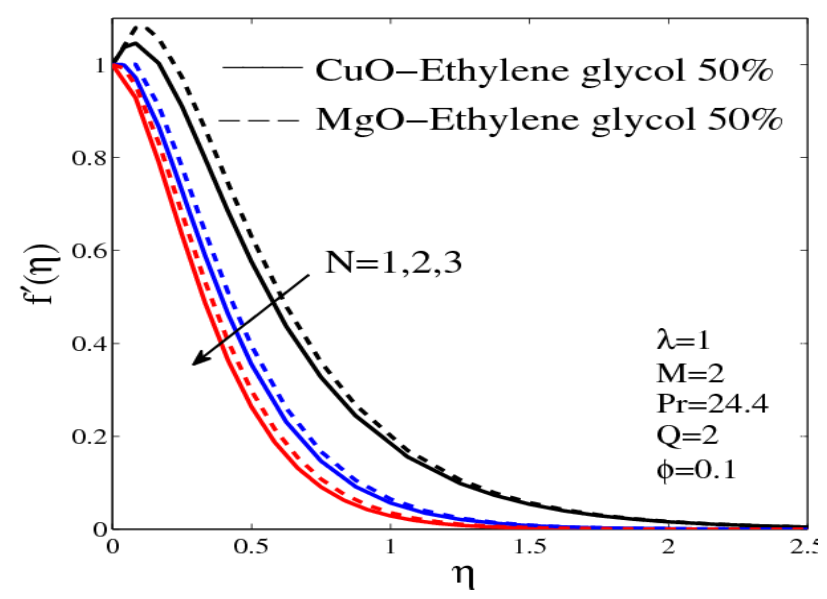

Fig. 6 Velocity profile for different $N$

In Fig. 6, the nanofluid velocity decreases with the increasing values of radiation parameter $N$. It is noted that the momentum of boundary layer thickness decreases when $N$ tends to decrease inside a boundary layer region and consequently it accelerates the viscosity of the nanofluid. Physically, the presence of thermal radiative parameter implies to more heat absorbs the liquid that compares to low velocity. $\mathrm{CuO}$ - ethylene glycol $50 \%$ is greater than that of $\mathrm{MgO}$ - ethylene glycol $50 \%$ owing to high thermal conductivity than magnesium oxide. 


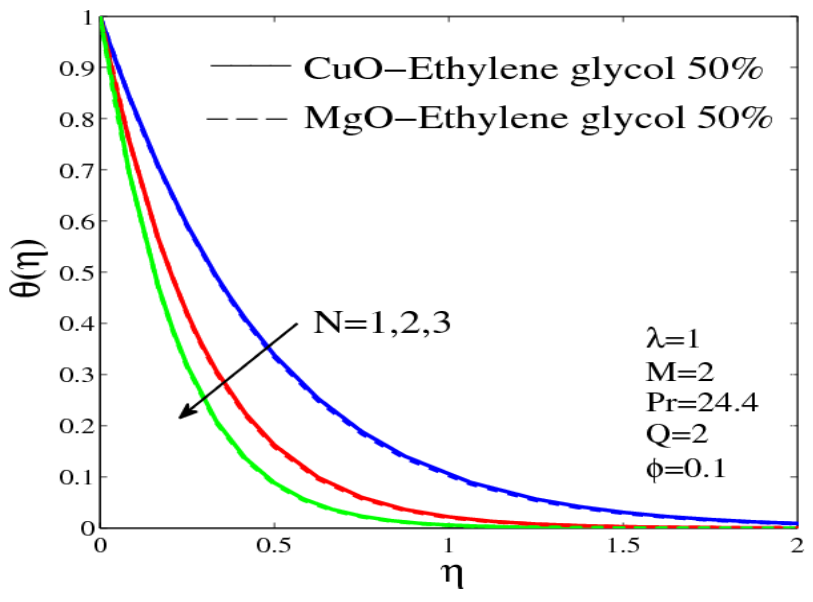

Fig. 7 Temperature for different $N$

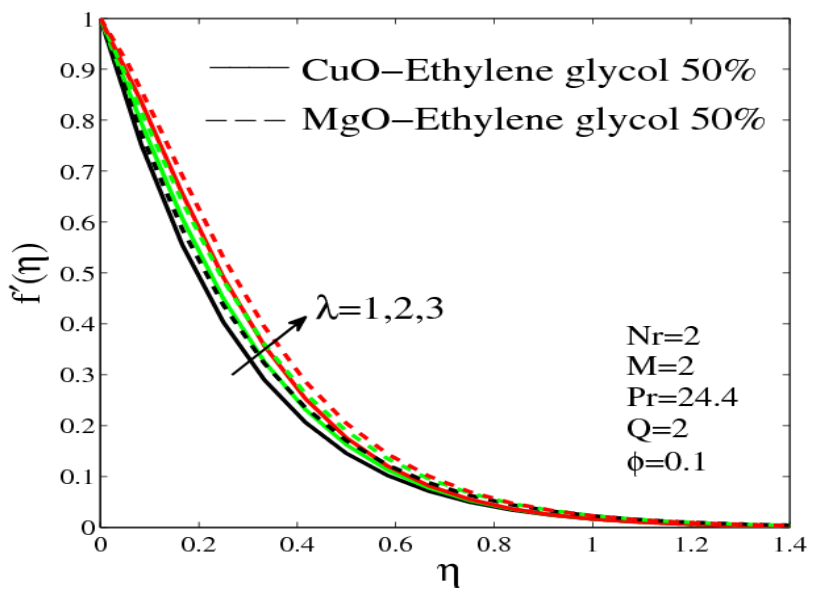

Fig. 8 Velocity profile for different $\lambda$

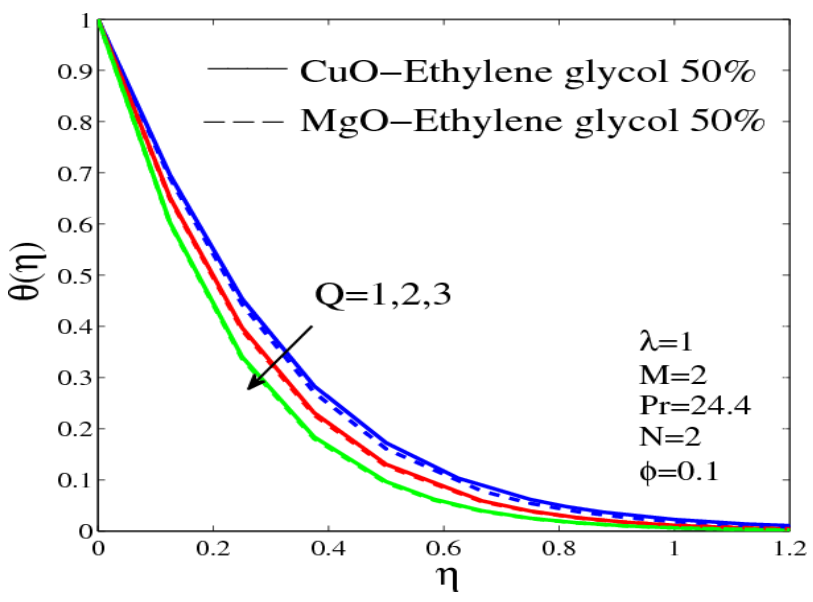

Fig. 9 Temperature profile for different $Q$

From Fig. 7 it is observed that the nanofluid temperature decreases as $N$ decreases; it is due to the fact that the conduction effect of the nanofluids decreases in the presence of thermal radiation. Therefore, higher values of radiation parameter incriminate higher surface heat flux and so, decrease the temperature within the boundary layer region, this leads to an increase in the heat transfer rate. The radiation parameter of $\mathrm{CuO}$ - ethylene glycol $50 \%$ are decreasing slower than that of MgOethylene glycol 50\% which can be attributed to the high thermal conductivity of copper oxide than magnesium oxide. For large values of $\mathrm{R}$, it is pointed out that, the temperature decreases more rapidly with the increase of $\mathrm{R}$, therefore, using radiation we can control the temperature distribution and flow transport, these types of applications can be used in pseudoscientific alternative medicine to control blood pressure through the process of magneto therapy.

From Fig. 8 depicts that the velocity profiles for $\mathrm{CuO}$ - ethylene glycol $50 \%$ and $\mathrm{MgO}$-ethylene glycol $50 \%$. Since the momentum boundary layer thickness increases with increasing values of buoyancy parameter enabling more flow. The effect of heat source parameter on the temperature field is shown in Fig. 9. It is noticed that the temperature of the fluid decreases with different values of heat source parameter. In the case of heat absorption, the absorbed heat by the nanofluid causes to decrease of more number of energy levels. Thus, decreases the temperature of the nanofluid.

\section{CONCLUSION}

Investigation of the MHD natural convective boundary-layer flow of a nanofluid and heat transfer over a stretching sheet considering the thermal radiation and heat source effects was represented in this paper. The resulting system of nonlinear partial differential equations is solved numerically by shooting method with Runge-Kutta fourth order scheme (MATLAB package). The effects of various parameters on velocity and temperature profiles are studied through graphs and table. The following conclusions have arrived:

- The velocity and temperature of the nanofluid increases with increasing of volume fraction parameter.

- The nanofluid velocity decreases as the existence of the magnetic field parameter becomes stronger

- The velocity and temperature of the nanofluid decreases with increasing of radiation parameter.

- The temperature of the nanofluid decreases with increasing of heat absorption parameter.

The present study has numerous applications involving heat transfer and other applications such as chemical sensors, biological applications, glass, solar energy transformation, electronics, petrochemical products, light-weight, heat-insulating and refractory fiberboard and metallic ceramics etc.

\section{REFERENCES}

Buongiorno, J., Hu, W., 2005, "Nanofluid Coolants for Advanced Nuclear Power Plants," Proceedings of ICAPP05, Seoul.15-19.

Choi, S. U. S., 1995, "Enhancing Thermal Conductivity of Fluids with Nanoparticles Developments and Applications of Non-Newtonian Flows," ASME FED 231/MD, 66, 99-105.

Choi, S. U. S., Zhang, Z. G., Yu, W., Lockwood, F. E., Grulke, E. A., 2001, "Anomalous Thermal Conductivity Enhancement in Nanotube Suspensions," Applied Physics Letters, 79, 2252.

http://dx.doi.org/10.1063/1.1408272

Das, S. K., Putra, N., Thiesen, P., Roetzel, W., 2003, "Temperature Dependence of Thermal Conductivity Enhancement for Nanofluids," Journal of Heat Transfer, 125, 567-574. http://dx.doi.org/10.1115/1.1571080

Daniel, Y., 2015, "Presence of Heat Generation/Absorption on Boundary Layer Slip Flow of Nanofluid over a Porous Stretching Sheet," American Journal of Heat and Mass Transfer, 2(1), 15-30.

http://dx.doi.org/10.7726/ajhmt.2015.100 
Eastman, J. A., Choi, S. U. S., Li, S., Thompson, L. J., Lee, S., 1997, "Enhanced Thermal Conductivity through the Development of Nanofluids," in Komarneni, S., Parker, J. C., Wollenberger, H. J., (Eds.), Nanophase and Nanocomposite Materials II, Materials Research Society, Pittsburgh.

Eastman, J. A., Choi, S. U. S., Li, S., Yu, W., Thompson, L. J., 2000, "Anomalously Increased Effective Thermal Conductivities of Ethylene Glycol-based Nanofluids Containing Copper Nanoparticles," Applied Physics Letters, 78, 718-720.

Gireesha, B. J., Mahanthesh, B., Shivakumara, I. S., and Eshwarappa, K.M., 2016, "Melting Heat Transfer in Boundary Layer StagnationPoint Flow of Nanofluid Toward a Stretching Sheet with Induced Magnetic Field," Engineering Science and Technology, 19(1), 313-321. http://dx.doi.org/10.1016/i.jestch.2015.07.012

Govindaraju, M., Vishnu Ganesh, N., Ganga, B., Abdul Hakeem, A. K., 2015, "Entropy Generation Analysis of Magneto Hydrodynamic Flow of a Nanofluid over a Stretching Sheet," Journal of the Egyptian Mathematical Society, 23(2), 429-434.

http://dx.doi.org/10.1016/j.joems.2014.04.005

Hamad, M. A. A., Ferdows, M., 2012, "Similarity Solutions to Viscous Flow and Heat Transfer of Nanofluid over Nonlinearly Stretching Sheet," Applied Mathematics and Mechanics English Edition, 33(7), 923-930.

http://dx.doi.org/10.1007/s10483-012-1595-7

Hamad, M.A.A., 2011, "Analytical Solution of Natural Convection Flow of a Nanofluid over a linearly Stretching Sheet in the Presence of Magnetic Field," International Communications in Heat and Mass Transfer, 38(4), 487-492.

http://dx.doi.org/10.1016/j.icheatmasstransfer.2010.12.042

Hemalatha, T., Akilandeswari, S., 2016, "Effect of Poly Ethylene Glycol on $\mathrm{CuO}$ Nanoparticles and its Antibacterial Application," International Letters of Chemistry, Physics and Astronomy, 63,111-118. http://dx.doi.org/10.18052/www.scipress.com/ilcpa.63.111

Hakan, F., Eiyad Abu-Nada, O., 2008, "Numerical study of natural convection in partially heated rectangular enclosures filled with nanofluids," International Journal of Heat and Fluid Flow, 29(5), 13261336.

http://dx.doi.org/10.1016/j.ijheatfluidflow.2008.04.009

Hayat, T., Imtiaz, M., Alsaed, A., 2014, "MHD Flow of Nanofluid over Permeable Stretching Sheet with Convective Boundary Conditions," Thermal Science, 139-139.

http://dx.doi.org/10.2298/tsci140819139h

Kakac, S., Pramuanjaroenkij, A., 2009, "Review of Convective Heat Transfer Enhancement with Nanofluids," International Journal of Heat Mass Transfer, 52, 3187-3196.

http://dx.doi.org/10.1016/j.ijheatmasstransfer.2009.02.006

Khan, W. A., Pop, I., 2010, "Boundary-layer Flow of a Nanofluid Past a Stretching Sheet," International Journal of Heat and Mass Transfer, 53(11-12), 477-2483. http://dx.doi.org/10.1016/j.ijheatmasstransfer.2010.01.032

Khan, M. S., Alam, M. M., Ferdows, M, 2013, "Effects of Magnetic Field on Radiative Flow of a Nanofluid Past a Stretching Sheet," Procedia Engineering, 56, 316-322,

http://dx.doi.org/10.1016/j.proeng.2013.03.125
Keblinski, P., Phillpot, S. P., Choi, S. U. S., Eastman, J. A., 2002 "Mechanism of Heat Flow is Suspensions of Nano-sized Particles (nanofluids)," International Journal of Heat Mass Tranfer, 42, 855-863. http://doi: 10.1016/S0017-9310(01)00175-2

Loganathan, P., Vimala, C., 2013, "MHD Boundary Layer Flow of a Nanofluid over an Exponentially Stretching Sheet in the Presence of Radiation," Heat Transfer-Asian Research, 43(4), 321-331.

http://dx.doi.org/10.1002/htj.21077

Makinde, O. D., Aziz, A., 2011, "Boundary Layer Flow of a Nanofluid Past a Stretching Sheet with a Convective Boundary Condition," International Journal of Thermal Sciences, 50(7), 1326-1332. http://dx.doi.org/10.1016/j.ijthermalsci.2011.02.01

Mabood, F., Shateyi, S., Rashidi, M.M., Momoniat, E., and Freidoonimehr, N., 2016, "MHD Stagnation Point Flow Heat and Mass Transfer of Nanofluids in Porous Medium with Radiation Viscous Dissipation and Chemical Reaction," Advanced Powder Technology, 27(2), 742-748.

http://dx.doi.org/10.1016/j.apt.2016.02.033

Nadeem, S., U1 Haq, R., Khan, Z. H., 2014, "Heat Transfer Analysis of Water-based Nanofluid over an Exponentially Stretching Sheet," Alexandria Engineering Journal, 53(1), 219-224.

http://dx.doi.org/10.1016/j.aej.2013.11.003

Nayak, M.K., Dash, G.C., Singh, L.P., 2014, "Steady MHD Flow and Heat Transfer of a Third Grade Fluid in Wire Coating Analysis with Temperature Dependent Viscosity," International Journal of Heat and Mass Transfer, 79, 1087-1095.

https://doi.org/10.1016/j.ijheatmasstransfer.2014.08.057

Nayak, M.K., Dash, G.C., Singh, L.P., 2014, "Effect of Chemical Reaction on MHD Flow of a Visco-elastic Fluid through Porous Medium," Journal of Applied Analysis and Computation, 4(4), 367-381

Nayak, M.K., 2015, "Chemical Reaction Effect on MHD Viscoelastic Fluid over a Stretching Sheet through Porous Medium," Meccanica, 51(8), 1699-1711.

https://doi.org/10.1007/s11012-015-0329-3

Nayak, M.K., Dash, G.C., Singh, L.P., 2015, “Unsteady Radiative MHD Free Convective Flow and Mass Transfer of a Viscoelastic Fluid Past an Inclined Porous Plate," Arabian Journal for Science and Engineering, 40(11), 3029 -3039.

https://doi.org/10.1007/s13369-015-1805-8

Nayak, M.K., Akbar, N.S., Tripathi, D., Khan, Z.H., Pandey, V.S., 2017, "MHD 3D Free Convective Flow of Nanofluid over an Exponentially Stretching Sheet with Chemical Reaction," Advanced Powder Technology, 28 (9), 2159-2166.

https://doi.org/10.1016/j.apt.2017.05.022

Nayak, M.K., Akbar, N.S., Tripathi, D., Pandey, V.S., 2017, "Three Dimensional MHD Flow of Nanofluid over an Exponential Porous Stretching Sheet with Convective Boundary Conditions," Thermal Science and Engineering Progress https://doi.org/10.1016/j.tsep.2017.07.006

Pak, B. C., Young, I. C., 1998, "Hydrodynamic and Heat Transfer Study of Dispersed Fluids with Submicron metallic Oxide Particles," Experimental Heat Transfer, 11(2), 151-170. http://dx.doi.org/10.1080/08916159808946559 
Patel, H. E., Sundararajan, T., Pradeep, T., Dasgupta, A., Dasgupta, N., Das S. K., 2005, “A Micro-Convection Model for Thermal Conductivity of Nanofluids," Pramana journal of physics, 65(5), 863-869. http://dx.doi.org/10.1007/bf02704086

Pourmehran, O., Rahimi-Gorji, M., Ganji, D. D., 2016, "Heat Transfer and Flow Analysis of Nanofluid Flow Induced by a Stretching Sheet in the Presence of an External Magnetic Field," Journal of the Taiwan Institute of Chemical Engineers, 65, 162-171.

http://dx.doi.org/10.1016/j.jtice.2016.04.035

Rashidi, M. M., Vishnu Ganesh, N., Abdul Hakeem, A. K., Ganga, B., 2014, "Buoyancy Effect on MHD Flow of Nanofluid over a Stretching Sheet in the Presence of Thermal Radiation," Journal of Molecular Liquids, 198, 234-238.

http://dx.doi.org/10.1016/j.molliq.2014.06.037

Rushi Kumar, B., Sravan Kumar, T., Vijaya Kumar, A. G., 2015, "Thermal Diffusion and Radiation Effects on Unsteady Free Convection Flow in the Presence of Magnetic Field Fixed Relative to the Fluid or to the Plate," Journal of Frontiers in Heat and Mass Transfer, 6, 6-12. http://dx.doi.org/10.5098/hmt.6.12

Rosseland, S., 1931 Astrophysik und atom-theoretische Grundlagen, Springer-Verlag, Berlin.

Sheikholeslami, M., GorjiBandpy, M., Ellahi, R., Zeeshan, A., 2014, "Simulation of MHD CuO-water Nanofluid Flow and Convective Heat Transfer Considering Lorentz Forces," Journal of Magnetism and Magnetic Materials, 369, 69-80.

http://dx.doi.org/10.1016/j.jmmm.2014.06.017

Sheikholeslami, M., Bandpy, M.G., Ellahi, R., Zeeshan, A., 2014, "Simulation of MHD CuO-Water Nanofluid Flow and Convective Heat Transfer Considering Lorentz Forces," Journal of Magnetism and Magnetic Materials, 369, 69-80. http://dx.doi.org/10.1016/i.jmmm.2014.06.017

Sheikholeslami, M., Ganji, D. D., 2014, "Unsteady Nanofluid Flow and Heat Transfer in Presence of Magnetic Field Considering Thermal Radiation," Journal of the Brazilian Society of Mechanical Sciences and Engineering, 37, 895 - 902.

http://dx.doi.org/10.1007/s40430-014-0228-x

Sekulić, J. E. ten Elshof, Dave H. A. B., 2005, “Selective Pervaporation of Water through a Nonselective Micro-porous Titania Membrane by a Dynamically Induced Molecular Sieving Mechanism," Langmuir, 21(2), $508-510$.

http://dx.doi.org/10.1021/la047458p

Satya Narayana, P. V., Venkateswarlu, B., 2016, "Heat and Mass Transfer on MHD Nanofluid Flow Past a Vertical Porous Plate in a Rotating System," Journal of Frontiers in Heat and Mass Transfer, 7, 110. https://doi.org/10.5098/hmt.7.8

Turkyilmazoglu, M., 2014, "Exact Analytical Solutions for Heat and Mass Transfer of MHD Slip Flow in Nanofluids," Chemical

Engineering Science, 84, 182-187.

http://dx.doi.org/10.1016/j.ces.2012.08.029

Xie, H. W. Y., Wei, C., 2010, "MgO Nanofluids: Higher Thermal Conductivity and Lower Viscosity among Ethylene Glycol-based Nanofluids Containing Oxide Nanoparticles," Journal of Experimental Nanoscience, 5(5), 463-472.

http://dx.doi.org/10.1080/17458081003628949

Yue, H., Zhao, Y., Ma, X., Gong, J., 2012, "Ethylene Glycol: Properties, Synthesis, and Applications," Chemical Society Reviews, 41(11), 4218. http://dx.doi.org/10.1039/c2cs15359a 